\title{
The Simulation Study Of Ultrasonic Signal From Multiple Measurement Points Based On The Radial Pipe
}

\author{
Wang Yueming, Wang Peng, Yao Guodong, Jia Hua,Li Wentao, Zhang Yaoyao \\ School of Information Engineering, Inner Mongolia University of Science and Technology, Baotou Inner \\ Mongolia, 014010, China
}

Keywords:ultrasonic,measurement,simulation,model,signal

\begin{abstract}
Gas-solid two-phase flow widely exists in industrial production. A simulation model is made by the finite element software, and is used to study the ultrasonic signals from multiple measurement points in the radial pipe. Through the research we found different voltage signals from the receiving end are obtained, when the measurement point is in different locations. The research provides some recommendations and technical proposals for the design of the ultrasonic sensor.
\end{abstract}

\section{Introduction}

The gas-solid two phase flow application is very popular with industrial production in the fields of medicine, chemistry, electricity, metallurgy, food, and so on. So it is studied by many experts and scholars.

Ultrasonic is a mechanical wave and its spread velocity is determined by medium largely. On account of the fact that ultrasonic is non-contacted measurement, it has become a research focus in fluid and some achievements have been made in recent years. Some ultrasonic measurement devices are mature in the United States, Germany, the Netherlands and so on. At home, some universities and research institutions have done some study in ultrasonic measuring instruments [4,5], and achieved a lot, such as in the measurement for velocity and attenuation of ultrasound in ultrafine particle suspensions[6,7]. As well, it is used in the measurement of the concentration of gas-solid two phase flow in recent years [8-9].

In this paper, a simulation model with finite element software COMSOL, is built to measure the signals from multiple measurement points in pipe. With building the simulation, the characteristics of signals from the different ultrasonic receiver are studied as to provide the reference for the placement of ultrasonic receiving devices. As a result, some advice and theory are suggested for designing the sensor to measure the gas-solid two phase flow.

\section{The simulation model of ultrasonic propagation in pipe}

Ultrasonic signals are spread in the actual fluid measurement. Simulation model is built with ultrasonic receiver distributing in a certain rule, in order to obtain the strength of the ultrasonic signals of different position in pipe. The results of the research will provide references for the installation site of the receiver.

\subsection{Introduction of the simulation model}

The simulation model of receivers in different locations is shown in Figure 1. In sub Figure (a), the pipe diameter is set to $\mathrm{R}$ (it is set to $300 \mathrm{~mm}$ under the actual working condition), and the section diameter of ultrasonic sensors is set to $0.05 \mathrm{R}$ $(\mathrm{d}=0.05 \mathrm{R})$. Sensors are composited by flexible compound piezoelectric materials $\mathrm{T}$ and $\mathrm{H}$, where the left upper is the transmitter (the end of $\mathrm{T}$ is shown in the figure), which is placed on arc $\mathrm{ABH}$. The receiver (it is $\mathrm{H}$ as shown in the figure) is on the other side. The sub Figure (b) is the simulation model meshed, where free partition is adopted here.

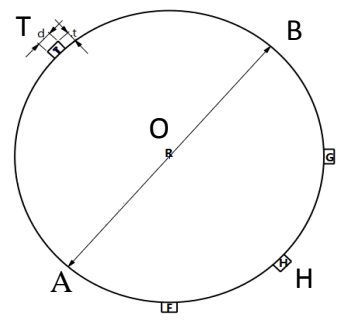

(a)

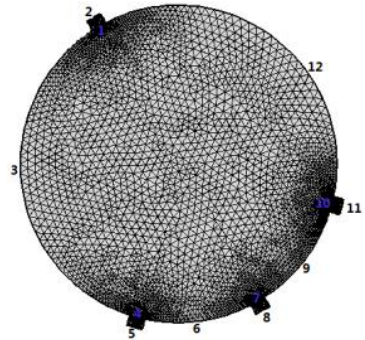

(b)
Figure 1. the COMSOL simulation model with three receivers in the pipe 
2.2 .The coupling equation of electricity - structure acoustic system

In this paper, finite element calculation mainly involve the electricity - structure - acoustic three physical field coupling, and the acoustic wave equation can be represented as:

$$
\frac{1}{\rho c^{2}} \frac{\partial^{2} p}{\partial t^{2}}+\nabla \cdot\left(-\frac{1}{\rho c}(\nabla p t)\right)=0
$$

where $\rho$ represents the density of material, c stands for the speed of sound, and $\nabla$ is Laplace operator, $p$ is the sound pressure. Structural mechanics equation can be expressed as:

$$
\rho \frac{\partial^{2} u}{\partial t^{2}}=\nabla \cdot s+F_{V}
$$

where $\rho$ is the density of piezoelectric material, $\mathrm{t}$ is the time, $\mathrm{u}$ represents displacement, $\mathrm{FV}$ is the volume force, and $\mathrm{s}$ is stress. Maxwell's equations of the Electric can be represented as:

$$
\nabla \cdot \mathrm{D}=\rho_{V}
$$

where $\rho_{V}$ represents the volume charge density, and D is the electric displacement.

In the simulation experiment, the inside boundary of compound piezoelectric material (PZT) $\mathrm{T}$ is set for the transmitter, and electrical boundary condition is as follows: instantaneous excitation voltage is $223.72 \mathrm{~V}$, whose equivalent circuit diagram is shown in Figure 2. The inside boundary condition on the compound piezoelectric of acoustic transmitting terminal $\mathrm{T}$ and receiver $\mathrm{H}$ is structure-acoustic coupling, and the electrical boundary condition on the outside for the piezoelectric material is grounded, its structural boundary condition is roller. Other boundary settings on the pipe wall are open.

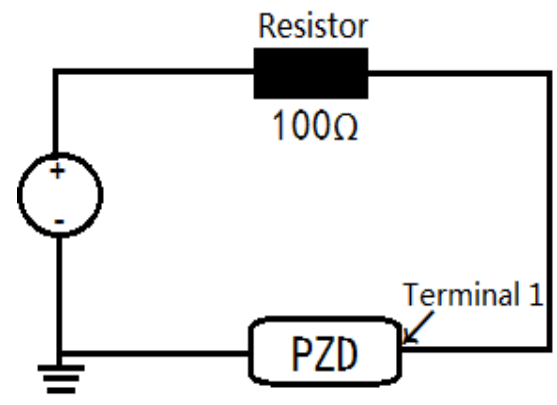

Fig 2 Equivalent circuit diagram of excitation voltage

\subsection{The experimental simulation}

In simulation experiment, in interior of the pipe is filled with air, Ultrasonic generator frequency is set to $30 \mathrm{KHZ}$. Record relevant experiment data,when the location of the ultrasonic receiver is changed.

In the arc $\mathrm{AHB}$ of the pipe, there is one receiver every time to analyze the voltage of the ultrasonic wave at different location of the receiver. The location of the receiver is set from the A to $\mathrm{H}$ finally to $\mathrm{B}$, with a measurement point (the ultrasonic receiver) every 15 degrees. There are 13 receiving points, which are point -6 , point -5 , point -4 , point -3 , point -2 , point -1 , point 0 , point 1 , point 2 , point 3 , point 4 , point 5 , point 6 (point 0 is $\mathrm{H}$ shown in Figure 1).

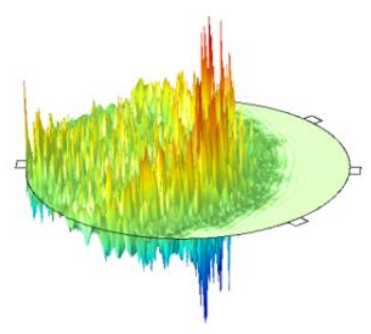

(a)

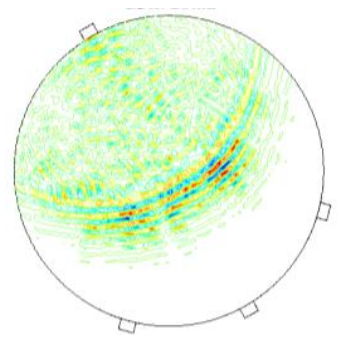

(b)
Fig 3 The simulation of sound pressure distribution in the pipe As is shown in Figure 3, it is the sound pressure distribution in the pipe when frequency is $30 \mathrm{KHZ}$, time is $5 \mathrm{e}-4 \mathrm{~s}$. Sub Figure (a) and sub Figure (b) are the stereo distribution map of pressure level and the equipotential map respectively. In the experiments, the maximum of voltage amplitude from receiver is gotten and saved, for collecting data at the receiving end respectively. Finally the analysis is been done that voltage amplitude is different at different receiving point.

\section{The results of simulation analysis}

It can be seen from the figure above that ultrasonic signals from different receiving point are different. Because the specific situation of the influence of ultrasonic on different point isn't still clear, then voltage amplitude at different receiving point is been analyzed. 


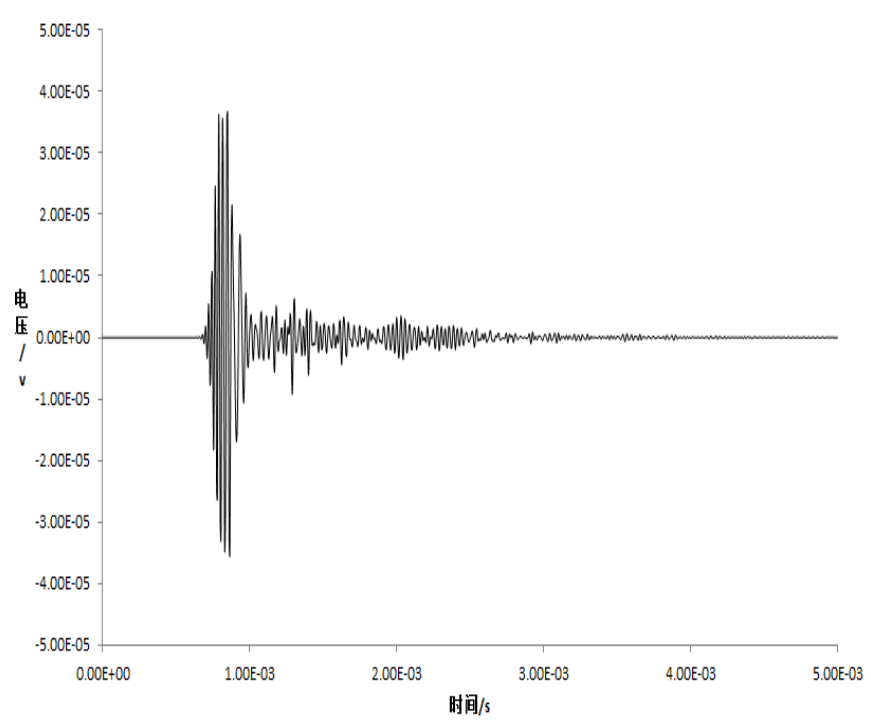

Fig 4 The receiver signal figure

As is shown in Figure 4, the receiver signals from different points are got in the experiment, but these figures are not on display here, in order to save the space. Signals received are symmetric disposition around the point $\mathrm{H}$, so that the average of the two symmetric points is seen as the final value. Figure 5 describes the signals received from different points, in which abscissa is the distance to the point $\mathrm{H}$ (such as the average of point -3 and point 3), ordinate represents the maximum amplitude of voltage signal (the unit for volt). It is seen from the figure that the received signal is the biggest of all with the ultrasonic receiver and transmitter in one direction, the received signal began to decay rapidly with the direction deviating from the transmitter and the impact of the distance between the transmitter and receiver to signals received is very little. When transmitter and receiver deviate in a certain extent, the change of attenuation will be slow (such as point 4, point 5 and point 6). The simulation experiment shows that the sensor should be placed on the emission direction or close to the emission direction in the actual sensors design of multiple detecting points.

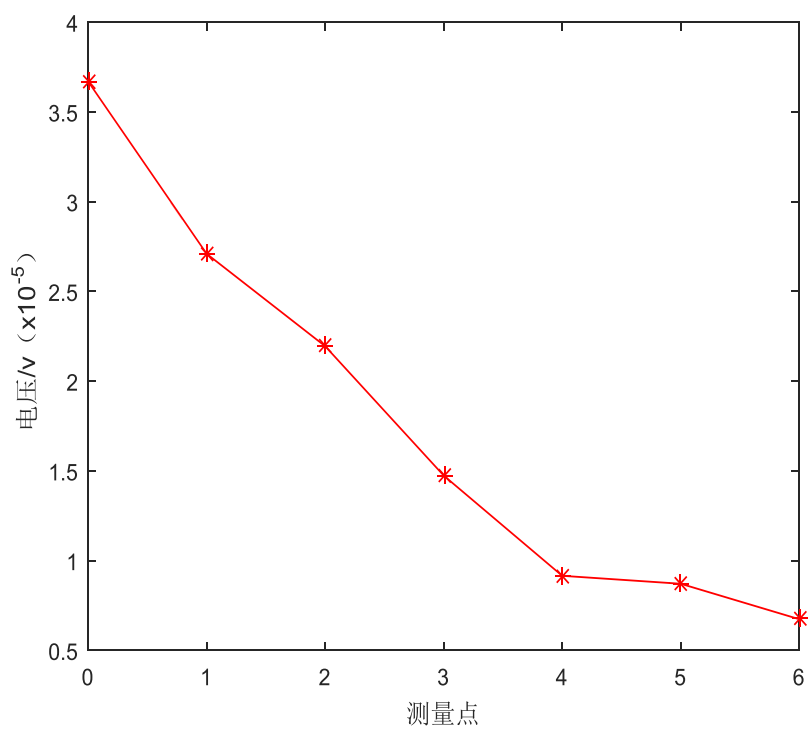

Fig 5 the signals from different points

\section{Conclusion}

In the paper, the impact of different points to signals received is been analyzed with building a simulation about ultrasonic propagation on the cross section of the pipe. The relationship between receiver points and the signals received gotten from the simulation provides some recommendations and technical proposals for the design of the ultrasonic sensor.

\section{Acknowledgments}

This work is supported by the National Natural Science Foundation of China(61463042) and Natural Science Foundation of Inner Mongolia(2014ms0609, 2015MS0549, 2016MS0611).

\section{Reference}

[1] SUN Jian-guo, JIN Ying-zi, JIN Xi-dong. Research on Numerical Simulation of Supersonic Gas-Solid Flow under Different Pressure Rations. Journal of Zhejiang Sci-Tech University,30(3):354-357, (2013).

[2] TIAN Chang ,SU Ming-Xu,CAI Xiao-Shu. Particle Concentration Characterization in Gas-Solid Two-Phase Flow by Ultrasonic Methods. Journal of Engineering Thermo physics, 34(8):1487-1490, (2013).

[3] F R M Yunus, R A Rahim. Simulation study of electrode size in air-bubble detection for dual-mode integrated electrical 
resistance and ultrasonic transmission tomography. Powder Technology,238 (2) :345-356,(2014).

[4] LI Qing, JIA Jing-pu. Design and Study of Ultrasonic Flow meter for High-temperature Flow Measurement. Coal Technology.31(1):209-211,( 2012).

[5] BAO Li-sha, LIANG Jun-ting, LU Jie. Mathematic model of multi-path ultrasonic gas flow meter. Technical Acoustics,29(4),393-395, (2010).

[6] SU Mingxu ,CAI Xiaoshu, The numerieal study of acoustical attenuation and veloeity in the suspension of superfine partieles. Acta Acustica;27(3):218-222, (2002).

[7] Hou Huaishu,Su Mingxu,Cai Xiaoshu. Study on measurement of ultrasonic attenuation and velocity spectra in superfine particle suspensions. Chinese Journal of Scientific Instrument;241-246, (2010). 